\title{
Event-building through Role-filling and Anaphora Resolution
}

\author{
Greg Whittemore \\ Electronic Data Systems Corp. \\ 5951 Jefferson Street N.E. \\ Albuquerque, NM 87109-3432 \\ greg@edsr.eds.com
}

\author{
Melissa Macpherson \\ Electronic Data Systems Corp. \\ 5951 Jefferson Street N.E. \\ Albuquerque, NM 87109-3432 \\ melissa@edsr.eds.com
}

\author{
Greg Carlson \\ Linguistics Program, University of Rochester \\ Rochester, NY \\ grca@uorvm.bitnet
}

\begin{abstract}
In this study we map out a way to build event representations incrementally, using information which may be widely distributed across a discourse. An enhanced Discourse Representation (Kamp, 1981) provides the vehicle both for carrying open event roles through the discourse until they can be instantiated by NPs, and for resolving the reference of these otherwise problematic NPs by binding them to the event roles.
\end{abstract}

\section{INTRODUCTION}

The computational linguistics literature includes a wide variety of ideas about how to represent events in as much detail as is required for reasoning about their implications. Less has been written about how to use information in text to incrementally build those event representations as discourse progresses, especially when the identification of event participants and other details is dispersed across a number of structures. We will be concerned here with providing a representational framework for this incremental event-building, and with using that representation to examine the ways in which reference to the internal structure of events contributes to discourse cohesion. That is, we will be interested both in the process of gleaning fully-specified event descriptions from continuous text, and in showing how individual elements of an event's internal structure can behave anaphorically.

Examples of the kinds of linkages that must be dealt with in building representations of events from text follow:

1a) He was believed to be a liar.

b) We promised him to be truthful. c) He tried to keep his mouth shut.

2a) Joe gave Pete a book to read.

b) Joe gave Pete a book to impress him.

c) Joe asked Pete for a book to read.

d) I asked Joe for a book to impress Sam.

e) Joe gave Pete the message to save his skin.

3a) Joe told Pete that to err is human.

b) He told us that to quit nould be silly.

4a) GI will broaden collaboration with Lotus to make a nen car.

b) Kary thought that an argument with herself would be entertaining.

c) Mary thought that a conference with himself would make John look silly.

The examples in (1) are familiar cases of syntactically obligatory control; we will consider their behavior to be straightforwardly and locally resolved. The sentences of (2) show infinitival relatives, purpose, and 'in-order-to' clauses in which control of the infinitive (and hence of its implicit subject) is sometimes clear, sometimes ambiguous. In (3), a subject infinitival phrase receives an unavoidably generic reading in one case and a non-generic but ambiguous reading in the other. Finally, the examples of (4) indicate that nominalizations of events also have roles whose reference must be determined, and whose existence and identity has consequences for subsequent discourse.

Aside from the sentences in (1), in which control is unambiguously sorted out within the sentence on the basis of verb type, all the examples above can 
be paraphrased with equivalent multi-sentence constructions in which the facts of referent-assignment are identical. Even more extended discourses, including dialogues such as that in (5), show the influence of an instantiated situation or event over the assignment of referents to entities introduced later in the discourse.

\section{5) A: John has been hobbling around for two veeks with a sprained ankle. \\ B: So what did the nurse say yesterday? \\ A : She said that it would not be smart to run so soon after injuring himself. \\ (adapted from Hishigauchi's 48, cited as a modification of Chao's 28)}

The distribution of event participants across multi-sentence discourses is sufficient to lay to rest any idea that the linkage is syntactically governed, even though the entities which provide cohesion in these examples are arguments which are typically bound syntactically. That is, it seems that initially unfilled thematic roles play a part in tying one sentence to the next. Event roles left unfilled after the operation of local syntactic processing are apparently still 'active', in some sense, and they appear to be able to attract participants from external structures to fill them. Carlson and Tanenhaus (1988) provide psycholinguistic evidence that this is indeed the case; open thematic roles do appear to be effective as cohesion devices. ${ }^{1}$

Previous theories about how open roles become filled (mostly intra-sententially) have been based on notions ranging from strictly syntactic to more pragmatic, knowledge-based approaches. Obviously wherever we do have what appears to be invariant and obligatory control, we want to exploit a syntactic explanation. However, these cases

\footnotetext{
${ }^{1}$ Whether it is just thematic roles, or those plus certain types of highly predictable adjuncts, or a wide variety of other types of slots which can provide the type of linking we are talking about is still an open question. We do assume that for each event we will encode not only THAT it expects certain arguments to be filled, but HOW it expects them to be filled; for instance it should be perceived that the noun 'salesman' is a highly suitable Agent for a sale event. We may need to know about more than that. In particular, we may require metonymical devices that make discourses like the following possible.

I had a hard time shopping.

First, the parking lot was all full...

Coherence in this example clearly depends on being able to associate 'the parking lot' with 'store' and 'store' with the Location of the 'shopping' event. This extension is no different in kind, however, from the core of what we are proposing here.
}

do not account for much of the ground that we need to cover. As the examples above show, even the syntactic position PRO often defies straightforward control assignment, and in the case of nominal references to events, Williams' (1985) arguments against a strictly syntactic account of referentassignment are convincing. Of course, there are no syntactic means for linking arguments with event descriptions intersententially. Appeals to underlying thematic role notions and/or more pragmatically governed operators then seem to hold more promise for the kinds of situations we are describing.

Given their currency above and below the sentence level, and the fact that they seem to be sensitive to both syntactic and pragmatic constraints, the behavior of unfilled event roles will best be explained at the discourse level. Like other discourse anaphoric elements, open roles can not only receive their reference from distant structures, but they also seem to be used productively to create links between linguistic structures and to extend focus in both forward and backward directions.

To machine-build representations of events whose essential components are dispersed across multiple structures, two key ingredients are necessary. First, the system must have knowledge about events and their expected participants and other characteristics. Given this, one can make predictions about the expectancy of arguments and the underlying properties they should hold. The second ingredient required is a means for assessing the mutual accessibility of discourse entities. As has been pointed out by various researchers, sentential structure, thematic relationships, and discourse configurations all may play a part in determining which entities must, might, and cannot be associated with others, and a discourse framework must make it possible to take all these factors into account in assigning reference and building representations of events.

Our intent in this paper is to provide a prototype model of event building which is effective across clauses, both intra- and inter-sententially. We will incorporate into this representation of events a means for assessing accessibility of events and event participants for anaphoric reference, and we will use the representation to examine the anaphoric behavior of open roles.

Event-Building Representation: We have chosen DRT as an overall representation scheme, though we will be modifying it to some extent. DRT has been designed to perform a variety of 
tasks, including proper placement of individual events in an overall discourse representation and making it possible to indicate which event entities are available for future anaphoric referencing and what constraints hold over those entities. A typical DR for a simple sentence is given in (6). The sentence, 'John gave Bill a dollar' is designated by the variable $\mathrm{E} 1$ and has associated with it a predicate calculus statement that contains the predicate, give, and argument variables V1, V2, and V3. The give event specification and other constraints, again in predicate calculus form, are contained in the lower portion of the DR. In the top half of the DR, any entities, including events, which are available for subsequent anaphoric referencing are listed by their variable names.

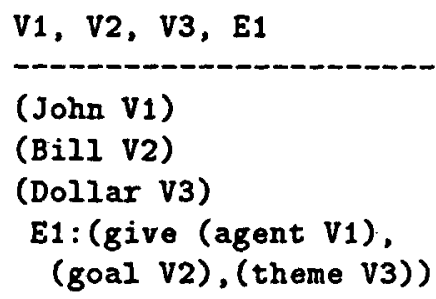

\section{A DR for John gave Bill a dollar.}

Our representation departs in some ways from the way in which the binding of anaphors is usually shown in DRT. In versions of DRT with realtime processing, whenever an NP is being processed, two things can happen: i) either the NP can be linked with a previously occurring NP and become anaphorically bound to it, or ii) a new referent can be generated for the NP and posted when no antecedent can be found. For our purposes, it is convenient to include in the DR an extra tier which contains items which have not yet found a referent. ${ }^{2}$ To designate the three parts of our DRs, we will use the following tier labels:

$$
\begin{aligned}
& \text { Available Referents - AR } \\
& \text { Unbound Referents - UR, and } \\
& \text { Constraints on Referents - CR. }
\end{aligned}
$$

For processing purposes, we will not attempt to immediately bind anaphors as they are encountered in sentences, beyond what we can get for free from syntactic analysis. Rather, we will initiate a twostage process, with the first DR having unbound anaphors and the second attempting representation of binding. In the first representation, we will

\footnotetext{
${ }^{2} \mathrm{~A}$ buffer of this sort may be implicit in other treatments of anaphora resolution; our extension is just to add it explicitly to the DR representation. Without some such buffer it is not clear how one would handle sentences like 'When he was a kid, John was pretty goofy.'
}

post unbound anaphors in UR. We will also post constraints for unbound items within CR to reflect their type, e.g. (PRO X1), (DEFINITE X2), and (HE X3). When items in UR become bound (or when their referents are found), their bindings will be represented in AR, they will be crossed off from within UR, and a new DR will be created to reflect the change in status.

We will also revise the representation of event descriptions in CR, by including in them implicit arguments for each event as well as ones which are explicitly realized in the sentence. Every event will have its underlying thematic and highly expected adjunctive roles posted in CR, whether the roles have been filled or not. These unfilled or implicit roles are posted as entities requiring binding, in UR. The constraint (IMPLICIT X) will be included for any open role, and for each event variable we will note in CR whether it was a verbal or otherthan-verbal description.

Example (7) contains an instance of what we intend. The nominalized form of an investigate event, marked with E1, has two open slots: Agent and Theme, V1 and V2, respectively. E1 is posted as a possible referent in AR; its two implicit arguments V1 and V2 are posted in UR. Similarly, E2, the launch event is posted in AR, while its open agent role, designated by V3, is shown in UR; its explicit Theme is already posted in AR as E1.

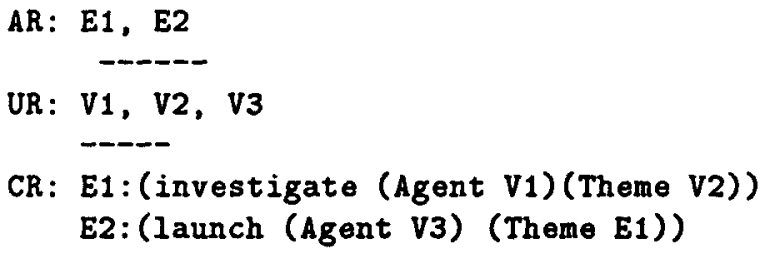

7. A DR of the sentence An investigation was launched.

We will show that because of the inclusion of open roles in the representation of events and on the UR tier, this framework for discourse representation makes it possible to link arguments that appear in a variety of structures to their respective events, and thus provides more predictive power for anaphoric resolution processes.

Verb-based Event References: We will demonstrate how DRs can be used to build interclausal events by providing various examples. We will move from the easiest examples, those that have much syntactic support, to the hardest, those whose resolution is mostly based on pragmatic grounds. 
We treat the binding of the PRO subject of embedded infinitive as a case of open role filling, and for our purposes, such binding is fundamentally the same in both obligatory and non-obligatory environments, since in every case the result is that open event roles are filled by arguments from external sources. That is, even where control is generated entirely within syntax, the links are construed as being the result of a cross-clause eventbuilding process. The operational difference is just that wherever control CAN be reliably determined syntactically, as in the case of obligatory control verbs, indices between controllers and PROs will be in place when initial DRs are generated. ${ }^{3} \mathrm{~A}$ typical DR with a controller-controllee relationship would appear as in (8).

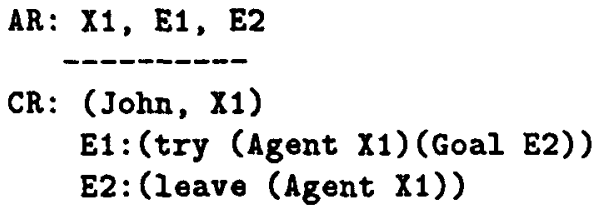

\section{The DR for John tried to leave.}

In the event-building examples that we show in the remainder of the paper, the aim is the construction of DRs that ultimately link events and arguments in this same way. What is different about the more complicated cases is just the means of accomplishing the linking. In the case of nonobligatory control of $\mathrm{PRO}$, such results may often require information from several levels of processing, and an adequate event-building representation must be able to accommodate the representation of all factors which are shown to be effective in predicting that control.

Nishigauchi (1984), for example, demonstrates that choice of controller can often be determined through knowledge of thematic roles (see also Bach, 1982, and Dowty and Ladusaw, 1988, for their accounts). In Nishigauchi's account, control of infinitival purpose clauses and infinitival relative clauses is primarily dependent on the presence of one of three thematic roles from his so-called Primary Location hierarchy; the idea is that a controller can be assigned if a Goal, Location, or Source is present in the sentence. Where a Goal is present, its refer-

\footnotetext{
${ }^{3}$ Dowty and Ladusaw (1988) believe that control is generally established via pragmatic means. They claim that it is pragmatic knowledge of events that enables one to generate links between participants and events. They also believe, however, that there are a large number of situations for which control has become grammaticized, and that there does not need to be any internal analysis in these situations to comprehend argument-to-event links.
}

ent has precedence as controller; where Goal is not present, Location or Source can take control.

The examples in (9) are indicative of the kinds of links that can be made via this hierarchy. In example (9a), the Goal 'Mary' controls the infinitival relative. ${ }^{4}$ In (9b), John ends up with the book, so 'John' is Goal, while in (9c), John as the possessor of the book is its Location; in both cases 'John' controls the infinitive.

(9)

a) John bought Mary a book PRO to read.

b) John bought a book PRO to read.

c) John has a book PRO to read.

To handle examples like (9a-c), we begin with initial DRs that include the kind of information that can be expected from a syntactic/semantic parser that produces initial logical forms. For instance, we know that 'John' is the Agent and 'Mary' the Goal of a buy event, and that the PRO subject of 'read' (the Agent of the read event) has no binding. The object of 'read' is identified in syntax as 'book'. An initial DR for (9a) is illustrated in (10).

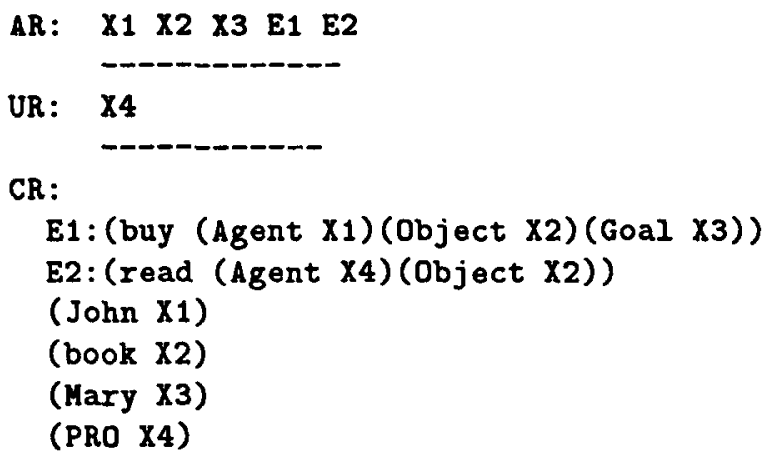

(10). The initial DR for John bought Mary a book to read.

At this stage, a positive check for Goal in E1 results in the binding of the unbound variable $\mathrm{X} 4$ to $\mathrm{X} 3$ in $\mathrm{AR} ; \mathrm{X} 4$ is then canceled out of UR. Were there no Goal in E1, a Location or Source would have the same effect. In a case where none of these roles is specified explicitly, as in example (11) (from Bach), it must be filled by default and/or from

\footnotetext{
4 'Mary' is more typically interpreted as Beneficiary in this sentence, but Nishigauchi claims that since Mary ends up with the book, she is the Goal. Bach's (1982) explanation is similar; it is that entity which the matrix verb puts in a position to do the VERBing which controls the infinitive.

${ }^{5}$ This analysis assumes that the infinitive is recognized as an infinitival relative on 'book', so that it does have an Object gap. The infinitive could also of course be an 'inorder-to' clause with intransitive 'read', in which case the controller is the Agent of 'buy'.
} 
context before it can bind the infinitive. In this case the default Goal for 'brought' is "present company", and so the PRO subject of 'enjoy' is first person plural inclusive.

\section{(11) I brought this miserable Morgon} to enjoy with our dinner.

Nominal Descriptions of Events: Much discussion has focused on the extent to which the internal structure of NPs that have nominalized events as heads, e.g. 'the destruction of the city by the Romans,' carries over the internal structure of the associated verb-headed structure, as in 'the Romans destroyed the city'. The consensus is that such deverbal noun phrases, while obviously semantically parallel in some ways, are not equivalent to verbal descriptions. In particular, semantic arguments associated with the nominalized form are held to be syntactically adjunctive in nature and entirely optional, even where they would be expressed as obligatory complements to the associated verb.

We are interested here in cases in which nominals representing events are linked with arguments that are not part of the same immediate syntactic environment. Several examples are provided in (12) and (13). As Higgins (1973, cf. Dowty, 1986) has discussed, in sentences like (12a) the subject of the matrix verb 'make' can be associated with the Agent position of an embedded nominal; therefore we understand 'Romans' to be the Agent of 'attack'. It is apparently the nature of the verb 'make' that permits this association; 'perform' behaves similarly. The verbs 'suffer' and 'undergo', on the other hand, link their subjects to the Theme or Experiencer of a nominalized event (that is, to what would be the expected object of the associated verb), as shown in (12b).

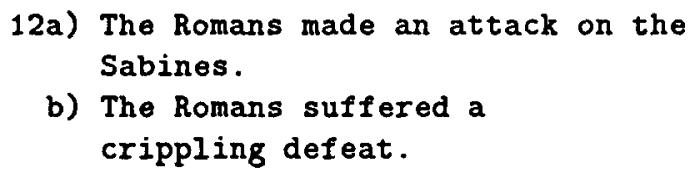

Williams (1985) makes use of the notion that a matrix verb can impose an association between its own arguments and any implicit arguments of a controlled event noun. However as the following examples show, not all verbs impose association of arguments to the degree that 'perform' and 'undergo' do. A verb may show some tendency toward association between Agents, as 'schedule' does in (13a), but be open to a realignment of matrix subject with some other more focused role in other environments, as in (13b). Some may have such a slight tendency to associate arguments in a particular way that it can be disrupted by syntactic structure, as in (13c) and (13d). In (13c) Sam may or may not be a party himself to the agreement, but in (13d) he is probably not involved.

(13a) John scheduled a takeover/meeting.

b) John scheduled a haircut/a checkup.

c) Sam negotiated an agreement.

d) An agreement ras negotiated by Sam.

What is necessary in order to sort this out is a working framework within which these tendencies can be represented and their interactions with other factors tracked. Where the tendency towards association is as strong as it is for 'make', which is considered to be semantically "bleached" in such constructions as make an attempt, make an arrangement, make a promise, make an attack (that is, it could be said to have become just a mechanism for linking matrix subject to object event), our representation will allow for an early linking at the level of syntax. For the general run of cases where an event noun is the object of a matrix verb, as in (13a-d), we must rely on our knowledge of typical interactions between events in order to decide what the linking between matrix subject and embedded event might be. The interaction between the $A R$ and the UR tiers of the DR, along with constraints on variables of both types, allows us to manipulate the association as may seem appropriate, with as much knowledge as we have at the time of linking.

Cross-Sentence Event-building: As we mentioned earlier, the linking phenomena we are examining hold across, as well as within sentences. Discourse (14) is provided as an example of a discourse in which an open role is filled in a subsequent sentence. In the first sentence, there are actually several open roles. Left unfilled are (at least) the roles Source and Exchange. With the DR structuring we have chosen, an initial DR for the first sentence of (14) would be built as in (15). The main thing to note in (15) is that the open role variables, are $\mathrm{Z1}$ and Q1, the Source and the Exchange, have been posted in UR.

(14a) Pete bought a car.

b) The salesman was a real jerk. 
(15)

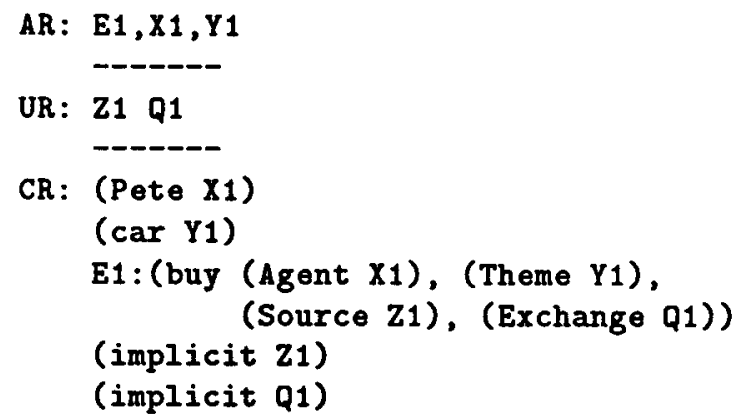

The initial DR for the second sentence of (14) is in (16a). The variable $\mathrm{X} 2$, representing 'the salesman', has been posted in the unresolved NP buffer, and $\mathrm{X} 2$ will be the first thing to be resolved by way of anaphora operators.

The anaphoric processes invoked at this point would be much like what has been promoted elsewhere. A variety of factors would come into play, including looking at basic semantic characteristics, centering, etc. We would also want to provide a means for ordering available referents as they are placed in AR in terms of their forward focusing character (Grosz, Joshi, and Weinstein, 1983).

For 'the salesman', the previously occurring discourse entities that are available as referents are E1, $\mathrm{X} 1$, and $\mathrm{Y} 1$ in the previous $\mathrm{AR}$, and $\mathrm{Z} 1$ and $\mathrm{Q} 1$ in the previous UR. The possible referent X1, 'Pete', ranks as a possible candidate but not a very likely one, since if Pete were to be referred to in a subsequent sentence it would more likely be done via a personal pronoun. The other available referent, Y1, the 'car', is semantically unlikely and is not considered a good choice. A search is then made into the previous UR. The Source Z1, in this instance, would be a highly likely choice, since any semantic qualities that would accompany 'the salesman' would fit those of the Source of a buy event.

It has been reported in previous studies that definite NPs often have no clear antecedent. For instance, 363 out of 649 definite NPs found in a study of corpus of dialogues (Brunner, Ferrara, and Whittemore, 1990) had no direct linguistic antecedents. $53 \%$ of the 363 definite NPs had semantically inferrable antecedents, where definite NPs were used to refer to attributes of antecedents and the like, but not to antecedents themselves. Apparently, definite NPs function to focus on some partial aspect of an antecedent or topic and not necessarily to refer directly to it as a whole. ${ }^{6}$ Following the

\footnotetext{
${ }^{6}$ The other $47 \%$ were reported to have no clear antecedents, and were only 'topically' tied to the context. It might prove beneficial to re-examine these true orphans and see if any of these refer back to open roles.
}

line of reasoning that one could take from these findings, it could be the case that there is actually a preference for definite NPs to refer back to open roles, since they represent particular points of focus or sub-components of events.

'Salesman', via the variable X2, would then get bound to the buy event and a second DR with no unresolved anaphora would be returned, as shown in $(16 b)$.

(16a)

AR: E2

$\mathrm{UR}: \mathrm{X2}$

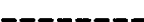

CR: (Salesman X2)

(definite $\times 2$ )

E2: (IS X2 real-jerk)

(16b)

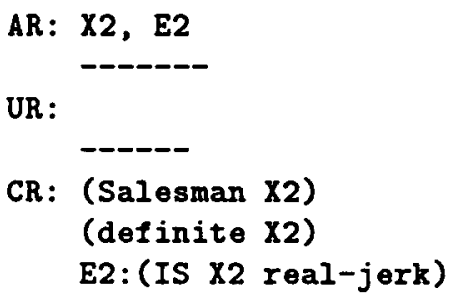

Similarly, the DR for the first sentence would need modification since now the open Source role, represented as $\mathrm{Z} 1$, would need to be bound to $\mathrm{X} 2$, 'the salesman' (this updated binding is not shown).

Limits on Linking: There are limits on the kinds of linking that can be effected between event descriptions and fillers for open roles. For instance, note that the open slot in the example above does not seem to be available for pronominal reference. If $(14 \mathrm{~b})$ is replaced with 'He was a real jerk,' the sequence of sentences makes no sense (or at least we would have to say that the same role is not accessed). This restriction appears to be true in general for pronominal reference into event descriptions, as the following examples show:

- I was attacked. ${ }^{*} \mathrm{He}$ was enormous.

- We unloaded the car. *They [the suitcases] were very heavy.

- This borrowing has got to stop. *They [the borrowed things] get left all over the place.

An event description itself, as a whole, nominal or verbal, may function as an antecedent for 
subsequent anaphoric reference, including pronominal reference ('I went swimming. It was horrible.'). It is just pronominal reference INTO an event description, especially a verbal one, which seems to be blocked. The event described in (17a) below cannot typically be elaborated upon by (17ai). However, (17ai) is fine as a continuation if (17aii), in which the event is nominalized, comes between. (17b), in which the agree event is referred to nominally, can be followed by (17bi), (17bii) or both.

(17)

a) Bob finally agreed with Joe.

i) *It was to not fight anymore.

ii) The agreement was negotiated by Sam.

b) Bob and Joe finally made an agreement.

i) It was to not $f$ ight anymore.

ii) It/The agreement was negotiated by Sam.

c) *It was between Bob and Sam.

In our representation the posting of event descriptions, verbal and nominal, in AR, accounts for the fact that each can be linked to by a subsequent pronominal element. Our intuition is that in order to be completely accessible as a referent, however, an entity must have not only a semantic but also a phonological realization; since open roles are merely implicit until they are bound, it is predictable that there would be a difference in their accessibility. For this reason we post open roles only in UR, not in AR, and in our framework this blocks pronominal access to them.

As for the fact that nominalizing an event seems to ease the restrictions on referring into it by means of a pronoun (as in the (17ai-ii) examples), our guess is that in these cases the pronominal reference is actually to the event as a thing, and that the apparent elaboration of roles is allowed by the same mechanisms that allow addition of other adjuncts to nominals, as in 'I really enjoyed my vacation. It was in Texas in July.' In any case our tagging of event variables in $C R$ as nominal or verbal allows this distinction to be taken into account.

The idea of role slots which are canceled from UR as they are bound explains another restriction on the ways in which events can be elaborated. (17c) above cannot appropriately follow either (17a) or (17b), because we already know from either that the agreement was between Bob and Joe. Further, if (17bii) follows (17b), then we know that Sam is not himself a participant in the agreement he negotiated, because we already know from (17b) that the agreement was between Bob and Joe. In each of these cases, the open role in question will have been canceled out of UR by binding to other entities before the new anaphoric elements come along, and so there is no possibility of filling a role twice.

Hard Cases: Finally, we offer a few comments on a "pretty hard" and a "really hard" example, given in (18) and (19). These are revised versions of the discourse given in (5). The task in both cases is to bind the referent 'John', which appears in the first sentence, to the Agent slot of 'run', which is in the second sentence.

(18)

John has been hobbling around on a sprained ankle.

Today, the nurse said it would be best not to run for two weeks.

(19)

John has been hobbling around on a sprained ankle.

Today, the nurse told his mother it would be best not to run for tro veeks.

To resolve these examples, we can employ two tactics. First, we will impose a thematic role association between the Addressee of a say event and the Agent of embedded agentless verbs that denote advice. Secondly, we will use the notion of open implicit roles in DRs to obtain a filler for the open Addressee role in the say/tell event. ${ }^{7}$

With these two notions in place, we can easily resolve (18). (18)'s context provides only one possible candidate for the open Addressee role, namely 'John' (that is, if we disregard the speaker of the utterance). Once 'John' is used to fill that role, we can link 'John also, through the default thematic role association, to the Agent slot for 'run'.

(19), however shows that the situation can be more complicated. There is no open Addressee role in (19); the explicit Addressee is 'his mother'. By the process above, then, 'his mother' would be linked to the Agent slot of 'run', which of course is incorrect. We do not have a perfect explanation for why (19) is different from (18), other than that John's mother is not the ultimate Addressee. That is, a mechanism is needed that can determine that John's mother transfers the advice on to the person who needs it, namely the ailing person, namely John. Even if such a complicated scenario is the

\footnotetext{
${ }^{7}$ A more general form of the first step would be a thematic role reasoning device that permits PROs to be linked with those entities which are most eligible to carry out the action of the subjectless infinitive. This formulation would be in the spirit of Bach, 1982.
} 
correct one, we believe that our combined thematic role/discourse representation would provide a platform upon which one could make use of such pragmatic information.

Conclusion: Our stated task was to provide a vehicle for constructing event representations which have roles that are not filled by local syntactic means. DRT is a natural vehicle for this kind of exercise, given certain extensions. The major extension is the posting of open event (thematic) roles as potential anchors for subsequent reference. In other words we are treating open roles as a type of anaphor. Where roles integral to an understanding of an event are not immediately filled on the basis of local syntax, we hypothesize that they should be posted nonetheless as not-yet-instantiated slots. We have added a tier to the conventional notion of a DR to accommodate this posting.

Our experiments with this representation have shown how information from various levels of processing can be brought together in event building. This framework also allows us to discover limits on linking phenomena; in particular, it naturally illustrates the inaccessibility of open roles to pronominal reference, and the tendency for definite NPs to link to substructures within an event.

\section{ACKNOWLEDGEMENTS}

We would like to note that the idea of using DRs as a means for building events across clauses came from a comment by Rich Thomason, cited in Dowty (1986:32): "Rich Thomason (p.c.) has suggested to me that a very natural way to construct a theory of event anaphora would be via Discourse Representation Theory." Thomason was addressing (we think) the notion of referring to events via nominalizations. We just extended the idea of using DRT to construct events across clauses to also include those denoted by verbs.

\section{References}

[1] Carlson, G. and Tanenhaus, M. 1988. Thematic Roles and Language Comprehension. THEMATIC RELATIONS, VOLUME 21 OF SYNTAX AND SEMANTICS, pgs. 263-291.

[2] Dowty, D. 1986. On the Semantic Content of the Notion "Thematic Role" . paper presented at the University of Massachusetts conference on Property Theory, Type Theory, and Semantics, March 13-16, 1986.
[3] Dowty, D. and Ladusaw, W. 1988. Toward a Nongrammatical Account of Thematic Roles, in Volume 21 of SYNTAX AND SEMANTICS, pgs. 61-73.

[4] Grosz, B., Joshi, A., and Weinstein, S. 1983. Providing a Unified Account of Definite Noun Phrases in Discourse. SRI Technical note \#292.

[5] Kamp, H. 1981. A Theory of Truth and Semantic Representation, in J. Groenendijk, $\mathbf{T}$. Jannsen, and M. Stokhof, (eds.). FORMAL METHODS IN THE STUDY OF LANGUAGE.

[6] Nishigauchi, T. 1984. Control and the Thematic Domain. LANGUAGE, Volume 60 , no. 2, pgs. 215-250.

[7] Williams, E. 1980. Predication. LINGUISTIC INQUIRY, Volume 11, no. 1, pgs. 203-238.

[8] Williams, E. 1985. PRO and Subject of NP. NATURAL LANGUAGE AND LINGUISTIC THEORY, Volume 3, no. 3, pgs. 297-315. 